\title{
Role of ERK1/2 activation on itch sensation induced by bradykinin $B 1$ activation in inflamed skin
}

\author{
YUANZHEN CHEN ${ }^{1,2^{*}}$, SHUYAN JIANG ${ }^{1,3^{*}}$, YUYING LIU ${ }^{1,2}$, \\ JIALING XIONG ${ }^{1,3}$, JIEXIAN LIANG ${ }^{1,3}$ and WENJIN JI ${ }^{2}$
}

\begin{abstract}
${ }^{1}$ Postgraduate Institute, Southern Medical University, Guangzhou, Guangdong 510515; ${ }^{2}$ Department of Anesthesiology;
${ }^{3}$ Division of Anesthesiology, Department of Cardiovascular Surgery, Guangdong Institute of Cardiovascular Diseases, Guangdong General Hospital, Guangdong Academy of Medical Sciences, Guangzhou, Guangdong 510080, P.R. China
\end{abstract}

Received February 11, 2015; Accepted April 11, 2016

DOI: $10.3892 / \mathrm{etm} .2016 .3426$

\begin{abstract}
It has previously been demonstrated that bradykinin receptor B1 (B1R) agonists evoke an itch-related scratching response in inflamed skin via the $\mathrm{B} 1$ receptor; however, the mechanisms responsible for this abnormal itch sensation remain unclear. Therefore, the present study utilized a complete Freund's adjuvant (CFA)-induced mouse model of inflammation to elucidate the mechanisms responsible. Over a period of $30 \mathrm{~min}$, scratching behavior was quantified by the number of hind limb scratches of the area surrounding the drug injection site on the neck. Furthermore, western blot analysis was used to investigate the potential role of extracellular signal-regulated kinase (ERK) 1/2 signaling as a mediator of itch in CFA-treated mice. The results demonstrated that CFA-induced inflammation at the back of the neck is associated with sustained enhancement of ERK1/2 activation in the spinal cord. Moreover, B1R agonist treatment resulted in increased expression of phosphorylated ERK1/2 in the spinal cord, which peaked at $45 \mathrm{~min}$. Consistent with these findings, inhibition of either mitogen-activated protein/ERK kinase or ERK1/2, as well as inhibition of ERK1/2 activation following inflammation, attenuated B1 receptor-mediated scratching responses to a
\end{abstract}

Correspondence to: Professor Jiexian Liang, Division of Anesthesiology, Department of Cardiovascular Surgery, Guangdong Institute of Cardiovascular Diseases, Guangdong General Hospital, Guangdong Academy of Medical Sciences, 96 DongChun Road, Guangzhou, Guangdong 510080, P.R. China

E-mail: jiwenjin@msn.com

Professor Wenjin Ji, Department of Anesthesiology, Guangdong General Hospital, Guangdong Academy of Medical Sciences, 96 DongChun Road, Guangzhou, Guangdong 510080, P.R. China E-mail: lijessy@msn.com

\section{${ }^{*}$ Contributed equally}

Key words: extracellular signal-regulated kinase 1/2, itch, kinin B1 receptor agonist, spinal cord, inflammation greater extent, as compared with control mice. Collectively, the results of the present study indicated that enhanced and persistent ERK1/2 activation in the spinal cord may be required to induce a scratching response to $\mathrm{B} 1 \mathrm{R}$ agonists following CFA-induced inflammation.

\section{Introduction}

Although itch, also known as alloknesis, and pain are unpleasant sensations, they are two distinct sensations (1); however, recent studies have identified various interactions between itch and pain transmission and sensitization pathways (2-4). For example, when painful stimuli is applied to lesional skin of patients with atopic dermatitis an itch sensation is evoked rather than pain, which is thought to be the result of central sensitization for itching $(5,6)$. Similarly, intradermal injection of algogenic substances capsaicin or bradykinin has been demonstrated to induce a scratching reflex, rather than pain-related behavior, in inflamed skin of mice $(7,8)$. The authors of the present study have previously reported that activation of bradykinin receptor B1 (B1R) contributes to alloknesis in mouse skin inflamed by treatment with complete Freund's adjuvant (CFA) (9). Moreover, B1R agonists are known to effectively induce scratch in inflamed lesioned skin (7). Although these previous studies have improved understanding of this phenomenon, the underlying mechanisms are yet to elucidated.

It has been well-documented that CFA is capable of activating ERK1/2 in primary sensory and secondary order dorsal horn neurons, and that pERK1/2 is associated with the generation and maintenance of inflammatory pain $(10,11)$. ERK1/2 activation has previously been described in the spinal cord during histamine- and DNFB-induced alloknesis (12), in DRG neurons during ET-1-induced acute alloknesis (13), during chronic alloknesis in dry skin and in allergic contact dermatitis models (14). However, its role in B1R agonist-induced alloknesis in an animal model of CFA inflammation has not been described. Therefore, we hypothesize that prolonged activation of ERK following CFA-induced inflammation may mediate the abnormal scratching response to B1R agonists. ERK1/2 are two closely related members of the mitogen-activated protein kinase family. Upon activation, phosphorylated 
ERK1/2 (pERK1/2) transduce extracellular stimuli into intracellular signaling and subsequently trigger the expression of a plethora of nuclear transcription factors to regulate numerous cellular functions $(15,16)$. Following peripheral nerve injury, phosphorylation and activation of ERK1/2 is induced in the dorsal root ganglia (DRG) (17-20) and dorsal horn of the spinal cord (21-23), and inhibition of ERK1/2 activation prevents behavioral pain sensitization $(11,24)$. At the molecular level, previous studies have demonstrated that activation of ERK1/2 signaling in the spinal cord or sensory neurons may be responsible for the relay of distinct types of stimulus-evoked acute itch and spontaneous scratching responses in chronic itch models $(12,14,25)$. It has also been demonstrated that ERK1/2 activation is required at the spinal level for itch responses, which are induced by histamine or dinitrofluorobenzene (DNFB) (12). Furthermore, it has been reported that pruritogenic molecules, including endothelin 1 (ET-1) and cytokine interleukin 31 (IL-31), stimulated alloknesis in cultured primary sensory neurons and induced phosphorylation of ERK1/2, whereas inhibition of ERK1/2 activation blocked ET-1- and cytokine IL-31-induced scratching behavior in vivo $(13,25)$. When taken together, these findings demonstrate that ERK1/2 activation in the spinal cord or sensory neurons may have an important role in itch transmission.

To our knowledge, there is a lack of previous studies investigating the mechanisms mediating itch-related scratching in response to algesic chemical stimuli delivered to inflamed tissue in mice. The aim of the present study was to investigate the potential role of ERK1/2 signaling in B1R agonist-induced alloknesis, using a CFA-induced mouse model of inflammation.

\section{Materials and methods}

Reagents and antibodies. CFA and MEK1/2 inhibitor (PD0325901) were purchased from Sigma-Aldrich (St. Louis, MO, USA). ERK1/2 inhibitor (328006) and kinin B1 receptor agonist [des-Arg(9)-bradykinin] were purchased from EMD Millipore (Billerica, MA, USA) and Tocris Bioscience (Bristol, UK), respectively. Rabbit anti-pERK1/2 (cat. no. 4370) and anti-ERK1/2 (cat. no. 9102) monoclonal antibodies, goat anti-rabbit horseradish peroxidase (HRP)-conjugated secondary antibody (cat. no. 7074) and rabbit anti-tubulin monoclonal antibody (cat. no. 2128) were obtained from Cell Signaling Technology, Inc., (Danvers, MA, USA).

Mice. A total of 98 male C57BL/6J mice, weighing 20-22 g and aged $\sim 6$ weeks, were obtained from the Center for Laboratory Animals, Sun Yat-Sen University (Guangzhou, China). Mice were maintained at $22 \pm 1^{\circ} \mathrm{C}$ on a $12 / 12 \mathrm{~h}$ light/dark cycle with ad libitum access to food and water. A mouse model of skin inflammation was established via intradermal injection of $50 \mu \mathrm{l}$ CFA into the nape of the neck, as previously described $(7,8)$. In the control group, an identical volume of normal saline was administered instead of CFA. The experimental procedures and animal use and care protocols were approved by the Committee on Ethical Use of Animals at the Guangdong Academy of Medical Sciences
(Guangzhou, China), and were performed according to the National Institutes of Health guidelines for the care and use of animals.

Behavioral tests. Seven mice from each of the CFA and control groups were used for the behavioral tests. Behavioral studies were conducted at approximately the same time each day between 9:00 a.m. and 4:00 p.m. in order to reduce circadian effects. Four days after injection with CFA, the mice were placed into individual small plastic chambers $(22 \times 12 \times 10 \mathrm{~cm})$ for $30 \mathrm{~min}$ prior to the experiment for acclimation. From video recordings over a period of $30 \mathrm{~min}$, scratching behavior was quantified by the number of hind limb scratches directed to the area surrounding the drug injection site on the neck. Off-site scratches, such as to the cheek, were excluded from the counts.

Treatment with BIR agonist. As peripheral noxious stimuli is capable of inducing phosphorylation of ERK1/2 $(26,27)$, western blot analysis was used to determine whether increased expression of pERK1/2 could be induced in the spinal cord by stimulation with a B1R agonist. Four days after inflammation was induced with CFA, mice $(n=15)$ were injected with des-Arg(9)-bradykinin B1R agonist $(0.4 \mathrm{mmol} / \mathrm{l})$ in the nape of the neck. The control group $(n=15)$ were administered normal saline stead of the B1R agonist. Mice were anesthetized with $1 \%$ sevoflurane (Wanshi Company, Osaka, Japan) and sacrificed by cervical dislocation at various time points $(5,15,30,45$ and $60 \mathrm{~min})$ post-injection. These time points were selected as they correspond with the time-dependent induction of pERK $1 / 2$ expression in the spinal cord by histamine (12). Cervical spinal cord samples were collected and preserved at $-80^{\circ} \mathrm{C}$ for analysis of pERK $1 / 2$ levels by western blotting.

Treatment with ERK1/2 and MEK1/2 inhibitors. CFA-treated mice were divided into four groups, as follows: i) 328006 group, in which mice were intraperitoneally injected with $30 \mathrm{mg} / \mathrm{kg}$ ERK1/2 inhibitor (100 $\mu \mathrm{l} 328006$ in 10\% DMSO; n=7); ii) PD0325901 group, in which mice were intraperitoneally injected with $10 \mathrm{mg} / \mathrm{kg}$ MEK1/2 inhibitor (100 $\mu 1$ PD0325901 in 10\% DMSO; $\mathrm{n}=7$ ); and iii) control group, in which mice were administered an identical volume of $10 \%$ DMSO and normal saline into the nape of the neck $(\mathrm{n}=14)$. The mice were treated with 328006 , PD0325901 or vehicle 30 min prior to intradermal administration of the des-Arg(9)-bradykinin B1R agonist $(0.4 \mathrm{mmol} / \mathrm{l}$ in $50 \mu \mathrm{l})$ into the nape of the neck. Dosages of ERK1/2 and MEK1/2 inhibitors were calculated based the study conducted by Kido-Nakahara et al (13).

Western blotting. Mice ( $\mathrm{n}=3 /$ group) were anesthetized with sevoflurane and sacrificed by cervical dislocation. Cervical cord segments and DRG neurons were dissected and preserved at $-80^{\circ} \mathrm{C}$. Lysates of DRG neurons or spinal cord sections were prepared by homogenization in lysis buffer containing a protease inhibitor mixture (Roche Diagnostics, Basel, Switzerland) and a phosphatase inhibitor cocktail (Thermo Fisher Scientific Inc., Waltham, MA, USA). Cell debris was removed by centrifugation at 
A

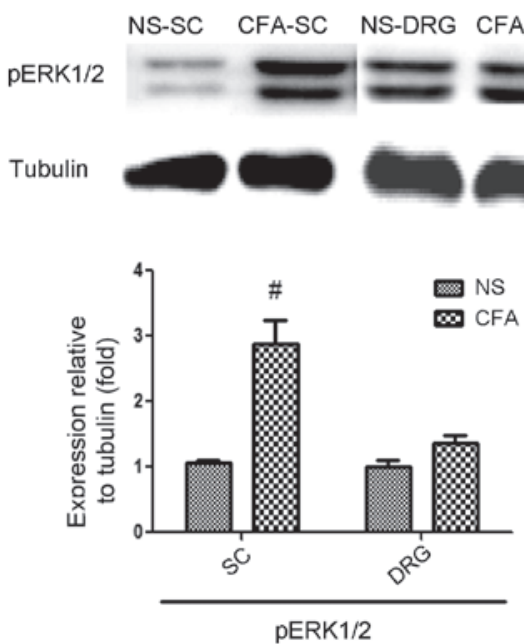

B

ERK1/2

CFA-SC CFA-DRG NS-SC NS-DRG

Tubulin
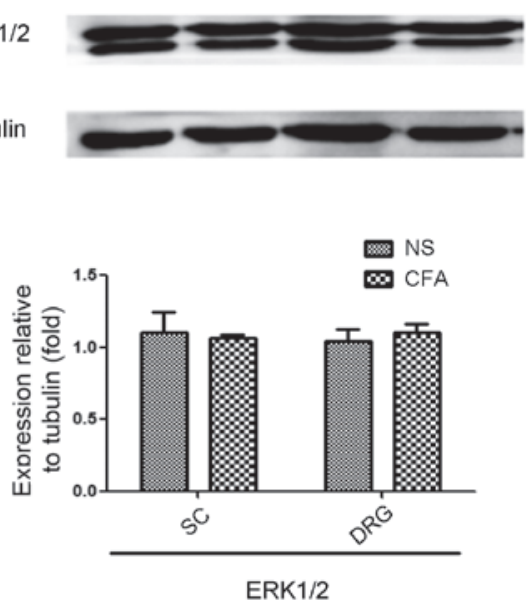

Figure 1. Increased level of pERK1/2 were detected in the spinal cord of CFA-induced mouse model of inflammation, as determined by western blot analysis. (A) Increased expression of pERK1/2 protein was detected in the spinal cord of CFA-inflamed mice, whereas pERK1/2 expression was unchanged in DRG neurons. (B) Invariable total ERK1/2 expression was detected in spinal cord and DRG neurons. Tublin was used as an internal reference. Data are presented as the mean \pm standard error of the mean. ${ }^{\text { }} \mathrm{P}<0.05$ vs. the NS group. pERK, phosphorylated extracellular signal-regulated kinase; SC, spinal cord; DRG, dorsal root ganglia; CFA, complete Freund's adjuvant; NS, normal saline.

$10,000 \mathrm{x} \mathrm{g}$ for $5 \mathrm{~min}$ at $4^{\circ} \mathrm{C}$. Samples were boiled for $5 \mathrm{~min}$ in sodium dodecyl sulfate (SDS) sample buffer, containing $1.0 \mathrm{~mol} / \mathrm{l}$ Tris-HCL, $8 \%$ SDS, $0.1 \%$ bromophenol blue, $10 \%$ glycerol and 2.5\% 2-mercaptoethanol (pH 6.8). Proteins were separated by SDS-polyacrylamide gel electrophoresis and transferred to polyvinylidene fluoride membranes (GE Healthcare Life Sciences, Chalfont, UK). Membranes were blocked with 5\% low-fat milk and incubated overnight at $4^{\circ} \mathrm{C}$ with rabbit anti-pERK1/2, anti-ERK1/2 $(1: 1,000)$ and anti-tubulin $(1: 2,000)$ monoclonal antibodies. Subsequently, the membranes were washed with phosphate-buffered saline and incubated with HRP-conjugated anti-rabbit secondary antibody $(1: 5,000)$ for $1 \mathrm{~h}$ at $37^{\circ} \mathrm{C}$. Labeled proteins were detected by incubation with enhanced chemiluminescence solution (Thermo Fisher Scientific, Inc.) for $1 \mathrm{~min}$ and visualized with an ImageQuant Las 4000 imager (GE Healthcare Biosciences, Pittsburgh, PA, USA). Tubulin was used as an internal control. Band intensities were quantified using ImageJ software, version 1.45 (https://imagej.nih.gov/ij/).

Statistical analysis. All results are expressed as the mean \pm standard error of the mean. Between-group comparisons of scratching responses were performed using unpaired t-tests. Two-factorial analysis of variance was used for all other comparisons. Statistical analyses were performed using SPSS software, version 19.0 (IBM SPSS, Armonk, NY, USA). $\mathrm{P}<0.05$ was considered to indicate a statistically significant difference.

\section{Results}

pERK1/2 expression levels are significantly increased in the cervical spinal cord of CFA-inflamed mice. To explore the potential role of ERK1/2 signaling as a mediator of alloknesis in CFA-treated mice, the present study investigated whether the expression levels of pERK1/2, which is a well-established indicator of ERK1/2 signaling activation, were increased in
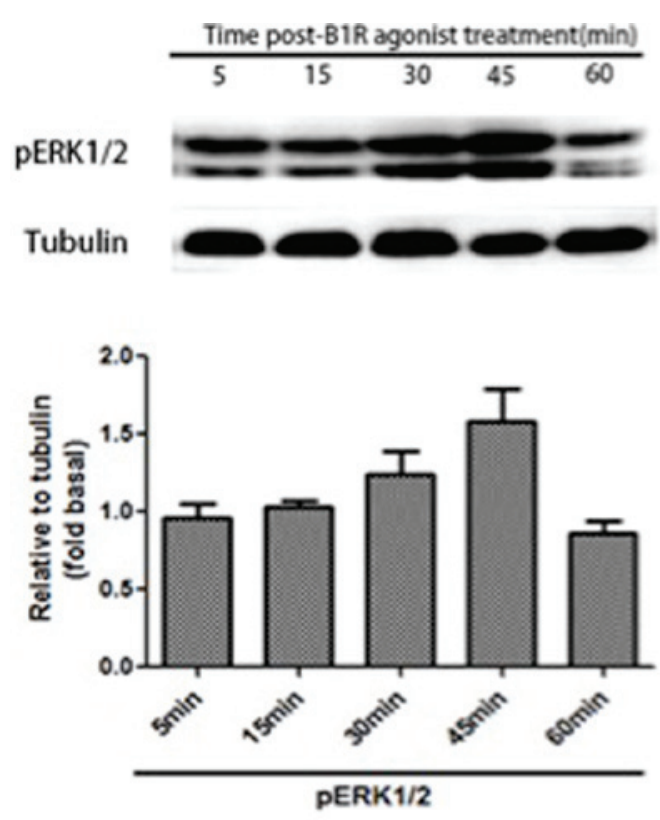

Figure 2. Time-dependent induction of pERK1/2 expression by B1R agonists in the spinal cord of CFA-inflamed mice. Following B1R agonist treatment, cervical spinal cords were harvested from CFA-inflamed mice at the specified time points and western blot analysis indicated that pERK1/2 is gradually upregulated from $30 \mathrm{~min}$ post-B1R agonist treatment, peaking at $45 \mathrm{~min}$ and subsequently declining to levels comparable with the NS control group after 60 min. Tublin was used as an internal control. pERK, phosphorylated extracellular signal-regulated kinase; B1R, bradykinin 1 receptor; CFA, complete Freund's adjuvant.

the spinal cord or DRG neurons of CFA-induced mice, as compared with control mice. Western blot analysis indicated that $\mathrm{pERK} 1 / 2$ were significantly increased in the cervical spinal cord 4 days after CFA-induced inflammation $(\mathrm{P}=0.0217)$, whereas pERK1/2 expression levels in DRG neuron extracts and total ERK1/2 levels were unchanged (Fig. 1). These results indicate that there was sustained ERK1/2 activation in the 


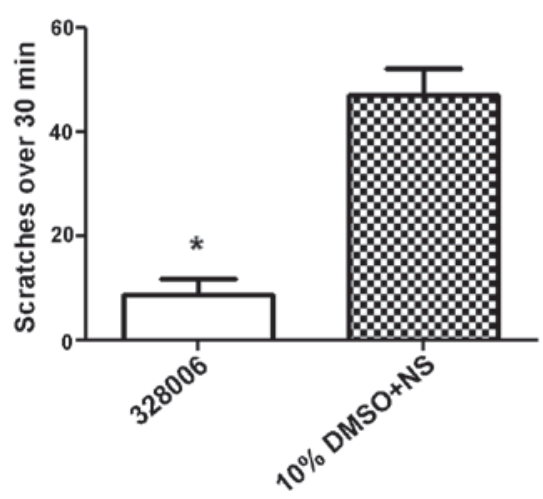

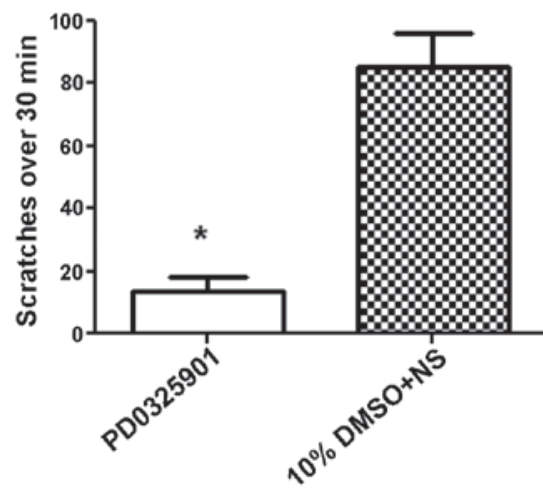

Figure 3. Blocking ERK1/2 signaling decreased B1R agonist-evoked scratching behavior in a mouse model of CFA-induced inflammation. (A and B) The number of scratches induced by B1R agonist treatment over a 30 min period was dramatically reduced by intraperitoneal injection of (A) PD0325901 MEK1/ERK1/2 inhibitor, which targets ERK1/2 phosphorylation and (B) 328006 ERK1/2 inhibitor, which targets ERK1/2, 30 min prior to intradermal bradykinin receptor B1 agonist injection into the nape of the neck. The respective control groups were administered $10 \%$ DMSO + NS. Data are presented as the mean \pm standard error of the mean $(n=7)$. ${ }^{*} \mathrm{P}<0.05$ vs. the control group. ERK, extracellular signal-regulated kinase; B1R, bradykinin 1 receptor; CFA, complete Freund's adjuvant inflamed mice; NS, normal saline.

spinal cord for at least 4 days following the injection of CFA in the nape region, which was not localized to DRG neurons.

pERK1/2 is upregulated following treatment with B1R agonist. As shown in Fig. 2, pERK1/2 expression levels in the cervical spinal cord increased 30 min post-B1R agonist administration, peaked at $45 \mathrm{~min}$, and then returned to control levels by 60 min post-administration. These results suggest that B1R agonist treatment is able to stimulate greater ERK1/2 activation in CFA-inflamed mice, as compared with the persistent activation induced by CFA treatment alone.

Inhibiting ERK1/2 signaling decreases B1R agonist-evoked scratching behavior. Western blot analysis indicated a potential role for ERK1/2 signaling in B1R agonist-induced pruritus in vivo. To further examine this hypothesis, a pharmacological approach was used to determine whether ERK1/2 activation is associated with the scratching response in vivo. PD0325901 MEK1/2 inhibitor, which targets ERK1/2 phosphorylation but is inactive against various other MAP kinase pathway kinases (28), was intraperitoneally administered $30 \mathrm{~min}$ prior to intradermal B1R agonist injection in the nape of neck. As compared with the control group, behavioral analyses demonstrated that B1R agonist-induced scratching behaviors were significantly reduced ( $>4$-fold) by inhibiting ERK1/2 via systemic application of the PD0325901 MEK1/2 inhibitor (P<0.001; Fig. 3A). Consistent with this result, treatment with the 328006 ERK1/2 inhibitor prior to B1R agonist intradermal injection also significantly decreased scratching (to a similar extent), as compared with the control group $(\mathrm{P}<0.001$; Fig. 3B). These results suggested that the ERK1/2 signaling pathway may be associated with the transmission of itch sensations in response to B1R agonist treatment in inflamed skin and indicated the contribution of ERK1/2 activation in the spinal cord as a mediator of this process.

\section{Discussion}

The present study demonstrated two major findings. Firstly, CFA-induced inflammation in the nape of the mouse neck produced a sustained increase in ERK1/2 activation in the cervical spinal cord, which was associated with increased scratching behavior in response to intradermal injection of the des-Arg(9)-bradykinin B1R agonist into the inflamed skin. Secondly, the upregulated levels of pERK1/2 in the CFA-induced mouse model of inflammation were further enhanced by treatment with the B1R agonist. This enhancement of pERK $1 / 2$ expression in the spinal cord may account for the abnormally elevated scratching behavior that was observed in response to B1R agonist treatment following CFA-induced inflammation.

Previous studies have demonstrated that ERK1/2 signaling in the spinal cord may contribute to the sensation of pain $(11,18,23)$. However, additional evidence has also suggested that the ERK1/2 signaling pathway is associated with itch transduction $(12,13,25)$. A previous study has demonstrated that pruritogens are capable of activating ERK1/2 signaling in the spinal cord, which is associated with the transduction of acute alloknesis (12). It has also been demonstrated that ERK1/2 phosphorylation in DRG neurons is critical for acute alloknesis, which are induced by cytokines IL-31 and ET-1 (13,25). Furthermore, ERK1/2 signaling in chronic alloknesis was also demonstrated by Zhao et al (14), who showed that pERK1/2 is a key mediator of itch sensations in the sensory neurons of mice with chronic alloknesis, since transient pERK1/2 activation in the spinal cord was absent when spontaneous scratching was observed. Therefore, these studies suggested that ERK1/2 activation in the spinal cord or DRG neurons is associated with neural transmission mediation of the itch sensation.

The present study investigated whether pERK1/2 expression increased in the spinal cord or DRG neurons $n$ response to B1R agonist stimulation during CFA-induced inflammation, and whether this increase was capable of mediating scratching behavior. The results of the present study demonstrated a persistent enhancement of pERK1/2 in the spinal cord of CFA-inflamed mice, but not in DRG neurons. These data are consistent with a recent study by Zhang et al (12), which showed that intradermal injection of pruritogen histamine into 
the nape or cheek of mice induced phosphorylation of ERK1/2 in the spinal cord but not in DRG neurons, and that ERK1/2 activation was crucial for the initiation and maintenance of the itch sensation. Conversely, our findings differ from a study published by Zhao et al (14), which indicated that persistent activation of ERK1/2 signaling in DRG neurons, but not in the spinal cord, was required for the maintenance of spontaneous scratching in allergic contact dermatitis and dry skin models of chronic alloknesis. It should be noted that scratching behaviour was detected in the present study, therefore the possibility that increases in pERK1/2 were elicited by the scratching itself and not by the pruritus produced by model treatments cannot be excluded. This may also account for the increased activation of ERK1/2 in DRG neurons that was observed in the chronic itch model $(12,29)$; whereas the present study demonstrated that enhanced levels of pERK1/2 were only required in the spinal cord for the B1R agonist-evoked itch sensation.

To elucidate whether B1R agonist treatment is capable of triggering alterations in the phosphorylation status of ERK1/2, similar to those observed following injection with histamine, the time-dependent induction of pERK $1 / 2$ in the cervical spinal cord was assessed in CFA-inflamed mice after B1R agonist injection $(9,30)$. Western blot analysis demonstrated that treatment with a B1R agonist induced further enhancement of ERK1/2 activation, which was already increased due to CFA inflammation. Furthermore, blocking ERK1/2 signal activation via systemic application of MEK1/2 or ERK1/2 inhibitors significantly decreased scratching behavior, indicating that the ERK1/2 signaling pathway may have a role in the itch sensation following B1R agonist application.

Phosphorylation of ERK1/2 in the spinal cord following peripheral inflammation by CFA contributes to persistent inflammatory pain $(10,21)$. The results of the present study suggest that activated ERK1/2 may have a role in the abnormal itch sensation following B1R activation in CFA-inflamed mice. It has previously been reported that the enhancement of nocifensive responses to metabotropic glutamate receptor agonist (RS)-3, 5-dihydroxyphenylglycine was associated with sustained ERK activation in dorsal horn neurons, which persisted for seven days following injection with CFA (31). It is possible that sustained ERK activation regulates plasticity in the spinal cord and underlies a component of central and peripheral sensitization, which leads to scratching in response to B1R agonist stimulation in CFA-inflamed skin $(32,33)$.

The results of the present study suggested that the persistence of ERK1/2 activation in the CFA inflammation model, and further upregulation by B1R agonist treatment in the spinal cord, underlies a change in the behavioral response to B1R activation in inflamed mice. Previous studies have demonstrated the involvement of B1R in pruriceptive processing following CFA-induced inflammation $(8,9)$ and B1R blockade has been shown to ameliorate CFA-induced inflammatory hyperalgesia $(34,35)$. A modulatory function for MAP kinase pathways has been demonstrated to regulate B1R expression during tissue injury (36) and control its function in inflammatory disorders (37). In addition, in previous studies B1R was upregulated in the airways of mice treated with TNF-a and IL-4, which also demonstrated the involvement of the MAPK pathway in this process $(38,39)$. Therefore, in light of these finding and the results of the present study, we hypothesize that there is an association between sustained ERK1/2 activation and B1R in CFA-inflamed mice. The present data suggested that the persistence of ERK1/2 activity itself is important for B1R agonist-mediated itch signaling following inflammation. However, it still remains to be investigated whether there is increased expression of B1R in the CFA-induced inflammation model and if sustained ERK1/2 activity upregulates B1R levels.

The authors of the present study have previously reported that a painful bradykinin stimulus evokes an itch sensation in CFA-inflamed skin, which was substantially blocked by a specific B1R antagonist (8). Therefore, CFA-induced inflammation may induce novel pruriceptive properties in B1R, resulting in B1R becoming responsive to algogenic stimuli. This previous study and the present results demonstrated that ERK1/2 activation in the spinal cord may be a prerequisite for the abnormal itch sensation stimulated by the B1R agonist during chronic inflammation. Therefore, the ERK1/2 signaling pathway may have a pivotal role in itch processing, providing novel insight into the molecular mechanisms that mediate itch responses to algogenic stimuli.

In conclusion, the present study demonstrated that CFA-induced inflammation results in persistent ERK1/2 activation in the spinal cord, which can be further upregulated by B1R agonist treatment, and is associated with itch processing in the spinal cord. As the cellular mechanisms that regulate itch sensations evoked by pain stimuli in inflamed skin remain poorly understood, further examination of the role of ERK1/2 signaling pathways in this process may yield novel mechanistic insights.

\section{Acknowledgements}

The present study was supported by the National Natural Science Foundation (grant nos. 81171040 and 81371236) and the Natural Science Foundation of Guangdong Province, China (grant no. 10151008002000005).

\section{References}

1. Ständer S and Schmelz M: Chronic itch and pain-Similarities and differences. Eur J Pain 10: 473, 2006.

2. Ma C, Nie H, Gu Q, Sikand P and Lamotte RH: In vivo responses of cutaneous $\mathrm{C}$-mechanosensitive neurons in mouse to punctate chemical stimuli that elicit itch and nociceptive sensations in humans. J Neurophysiol 107: 357-363, 2012.

3. Liu T and Ji R: New insights into the mechanisms of itch: Are pain and itch controlled by distinct mechanisms? Pflügers Arch 465: 1671-1685, 2013.

4. Davidson S and Giesler GJ: The multiple pathways for itch and their interactions with pain. Trends Neurosci 33: 550-558, 2010.

5. Hosogi M, Schmelz M, Miyachi Y and Ikoma A: Bradykinin is a potent pruritogen in atopic dermatitis: A switch from pain to itch. Pain 126: 16-23, 2006.

6. Sikand P, Shimada SG, Green BG and LaMotte RH: Similar itch and nociceptive sensations evoked by punctate cutaneous application of capsaicin, histamine and cowhage. Pain 144: 66-75, 2009.

7. Liang J, Xiao G and Ji W: Capsaicin induces reflex scratching in inflamed skin. Pharmacology 88: 82-87, 2011.

8. Liang J, He Y and Ji W: Bradykinin-evoked scratching responses in complete Freund's adjuvant-inflamed skin through activation of B1 receptor. Exp Biol Med (Maywood) 237: 318-326, 2012.

9. Feng J, Chen Y, Xiong J, Chen X, Liang J and Ji W: The kinin B1 receptor mediates alloknesis in a murine model of inflammation. Neurosci Lett 560: 31-35, 2014. 
10. Fang JQ, Fang JF, Liang Y and Du JY: Electroacupuncture mediates extracellular signal-regulated kinase $1 / 2$ pathways in the spinal cord of rats with inflammatory pain. BMC Complement Altern Med 14: 285, 2014.

11. Ji RR, Befort K, Brenner GJ and Woolf CJ: ERK MAP kinase activation in superficial spinal cord neurons induces prodynorphin and NK-1 upregulation and contributes to persistent inflammatory pain hypersensitivity. J Neurosci 22: 478-485, 2002.

12. Zhang L, Jiang GY, Song NJ, Huang Y, Chen JY, Wang QX and Ding YQ: Extracellular signal-regulated kinase (ERK) activation is required for itch sensation in the spinal cord. Mol Brain 7: 25, 2014.

13. Kido-Nakahara M, Buddenkotte J, Kempkes C, Ikoma A, Cevikbas F, Akiyama T, Nunes F, Seeliger S, Hasdemir B, Mess C, et al: Neural peptidase endothelin-converting enzyme 1 regulates endothelin 1-induced pruritus. J Clin Invest 124 2683-2695, 2014.

14. Zhao ZQ, Huo FQ, Jeffry J, Hampton L, Demehri S, Kim S, Liu XY, Barry DM, Wan L, Liu ZC, et al: Chronic itch development in sensory neurons requires BRAF signaling pathways. J Clin Invest 123: 4769-4780, 2013

15. Pearson G, Robinson F, Beers Gibson T, Xu BE, Karandikar M, Berman K and Cobb MH: Mitogen-activated protein (MAP) kinase pathways: Regulation and physiological functions. Endocr Rev 22: 153-183, 2001.

16. Shaul YD and Seger R: The MEK/ERK cascade: From signaling specificity to diverse functions. Biochim Biophys Acta 1773: 1213-1226, 2007.

17. Seino D, Tokunaga A, Tachibana T, Yoshiya S, Dai Y, Obata K, Yamanaka H, Kobayashi K and Noguchi K: The role of ERK signaling and the $\mathrm{P} 2 \mathrm{X}$ receptor on mechanical pain evoked by movement of inflamed knee joint. Pain 123: 193-203, 2006.

18. Obata K and Noguchi K: MAPK activation in nociceptive neurons and pain hypersensitivity. Life Sci 74: 2643-2653, 2004.

19. Obata K, Yamanaka H, Dai Y, Mizushima T, Fukuoka T, Tokunaga A and Noguchi K: Differential activation of MAPK in injured and uninjured DRG neurons following chronic constriction injury of the sciatic nerve in rats. Eur J Neurosci 20 : 2881-2895, 2004.

20. Obata K, Yamanaka H, Dai Y, Mizushima T, Fukuoka T, Tokunaga A and Noguchi K: Activation of extracellular signal-regulated protein kinase in the dorsal root ganglion following inflammation near the nerve cell body. Neuroscience 126: 1011-1021, 2004.

21. Obata K, Yamanaka H, Dai Y, Tachibana T, Fukuoka T, Tokunaga A, Yoshikawa H and Noguchi K: Differential activation of extracellular signal-regulated protein kinase in primary afferent neurons regulates brain-derived neurotrophic factor expression after peripheral inflammation and nerve injury. J Neurosci 23: 4117-4126, 2003.

22. Zhang X, Zhang H, Shao H, Xue Q and Yu B: ERK MAP kinase activation in spinal cord regulates phosphorylation of cdk5 at serine 159 and contributes to peripheral inflammation induced pain/hypersensitivity. PLoS One 9: e87788, 2014.

23. Zhang YL, Shi XD, Fu D, Xu JM and Dai RP: Activation of spinal ERK1/2 contributes to mechanical allodynia in a rat model of postoperative pain. Molecular Medicine Reports 7: 1661-1665, 2013.

24. Sanna MD, Mello T, Ghelardini C and Galeotti N: Inhibition of spinal ERK1/2-c-JUN signaling pathway counteracts the development of low doses morphine-induced hyperalgesia. Eur J Pharmacol 764: 271-277, 2015.
25. Cevikbas F, Wang X, Akiyama T, Kempkes C, Savinko T, Antal A, Kukova G, Buhl T, Ikoma A, Buddenkotte J, et al: A sensory neuron-expressed IL-31 receptor mediates T helper cell-dependent itch: Involvement of TRPV1 and TRPA1. J Allergy Clin Immunol 133: 448-460, 2014.

26. Ji RR, Baba H, Brenner GJ and Woolf CJ: Nociceptive-specific activation of ERK in spinal neurons contributes to pain hypersensitivity. Nat Neurosci 2: 1114-1119, 1999.

27. Karim F, Hu HJ, Adwanikar H, Kaplan D and Gereau RW IV: Impaired inflammatory pain and thermal hyperalgesia in mice expressing neuron-specific dominant negative mitogen activated protein kinase kinase (MEK). Mol Pain 2: 2, 2006.

28. Favata MF, Horiuchi KY, Manos EJ, Daulerio AJ, Stradley DA, Feeser WS, Van Dyk DE, Pitts WJ, Earl RA, Hobbs F, et al: Identification of a novel inhibitor of mitogen-activated protein kinase kinase. J Biol Chem 273: 18623-18632, 1998.

29. Song XS, Cao JL, Xu YB, He JH, Zhang LC and Zeng YM: Activation of ERK/CREB pathway in spinal cord contributes to chronic constrictive injury-induced neuropathic pain in rats. Acta Pharmacol Sin 26: 789-798, 2005.

30. Kilstein Y, Nowak W, Errasti AE, Feás AA, Armesto AR, Pelorosso FG and Rothlin RP: Involvement of Extracellular Signal-Regulated Kinase 5 in Kinin B1 Receptor Upregulation in Isolated Human Umbilical Veins. J Pharmacol Exp Ther 357: 114-124, 2016.

31. Adwanikar H, Karim F and Gereau RW IV: Inflammation persistently enhances nocifensive behaviors mediated by spinal group I mGluRs through sustained ERK activation. Pain 111: $125-135,2004$

32. Gao YJ and Ji RR: Light touch induces ERK activation in superficial dorsal horn neurons after inflammation: Involvement of spinal astrocytes and JNK signaling in touch-evoked central sensitization and mechanical allodynia. J Neurochem 115: 505-514, 2010.

33. Xu YM, Wang XT, Zhang ZY, Suo ZW, Yang X and Hu XD: Noradrenergic $\alpha 2$ receptor attenuated inflammatory pain through STEP61/ERK signalling. Eur J Pain 19: 1298-1307, 2015.

34. Ferreira J, Campos MM, Pesquero JB, Araujo RC, Bader M and Calixto JB: Evidence for the participation of kinins in Freund's adjuvant-induced inflammatory and nociceptive responses in kinin $\mathrm{B} 1$ and $\mathrm{B} 2$ receptor knockout mice. Neuropharmacology 41: 1006-1012, 2001.

35. Luo MC, Chen Q, Ossipov MH, Rankin DR, Porreca F and Lai J: Spinal dynorphin and bradykinin receptors maintain inflammatory hyperalgesia. J Pain 9: 1096-1105, 2008.

36. Larrivee JF, Bachvarov DR, Houle F, Landry J, Huot J and Marceau F: Role of the mitogen-activated protein kinases in the expression of the kinin B1 receptors induced by tissue injury. J Immunol 160: 1419-1426, 1998.

37. Ehrenfeld P, Matus CE, Pavicic F, Toledo C, Nualart F, Gonzalez CB, Burgos RA, Bhoola KD and Figueroa CD: Kinin B1receptor activation turns on exocytosis of matrix metalloprotease-9 and myeloperoxidase in human neutrophils: Involvement of mitogen-activated protein kinase family. J Leukoc Biol 86: 1179-1189, 2009.

38. Zhang Y, Adner M and Cardell LO: Up-regulation of bradykinin receptors in a murine in-vitro model of chronic airway inflammation. Eur J Pharmacol 489: 117-126, 2004.

39. Bryborn $M$, Adner $M$ and Cardell LO: Interleukin-4 increases murine airway response to kinins, via up-regulation of bradykinin B1-receptors and altered signalling along mitogen-activated protein kinase pathways. Clin Exp Allergy 34: 1291-1298, 2004. 